\title{
Magnoflorine promotes Huh-7 cell apoptosis and autophagy by regulating PI3K/Akt/mTOR pathway
}

\author{
Jifan $\mathrm{Xu}^{1}$, Bo Du${ }^{1}$, Yunfeng Liu ${ }^{1}$, Chonglin $\mathrm{Tao}^{2 *}$ \\ ${ }^{1}$ Department of Hepatobiliary Surgery, Peoples Hospital of Chongqing Kaizhou, Chongqing, China; ${ }^{2}$ Department of \\ Hepatopancreatobiliary Surgery, the First Affiliated Hospital of Wenzhou Medical University, Wenzhou, Zhejiang, China
}

*Corresponding Author: Chonglin Tao, Department of Hepatopancreatobiliary Surgery, The First Affiliated Hospital of Wenzhou Medical University, Shangcai Village, Baixiang Street, Ouhai District, Wenzhou, Zhejiang 325000, China. Email: cltao8185@163.com

Received: 23 November 2021; Accepted: 14 December 2021; Published: 20 January 2022

(c) 2022 Codon Publications

\section{OPEN ACCESS @(1)(?) ORIGINAL RESEARCH}

\begin{abstract}
Hepatoma is a malignant tumor with high rates of heterogeneity, metastasis, and mortality. Currently, there is no effective treatment available for hepatoma. In order to treat advanced hepatoma in a better manner, new and more effective therapeutic targets still need to be developed. Magnoflorine (MGN) is a quaternary ammonium alkaloid with a variety of therapeutic properties. MGN inhibited the proliferation of lung cancer, breast cancer, glioma, and rhabdomyosarcoma cells, induced apoptosis, and blocked cell cycle. However, its possible effects on the progression of hepatoma are still indefinite. In this study, the effects of MGN on the progression of hepatoma in vitro and the underlying mechanisms were determined. MGN suppressed the proliferation, induced the autophagy, and stimulated the apoptosis of human hepatoma Huh-7 cells. Mechanically, MGN could regulate PI3K/AKT/mTOR pathway, which therefore affects the progression of hepatoma in vitro. Taken together, MGN affected Huh-7 cell proliferation, autophagy, and apoptosis, and might act as a promising therapeutic drug for treating hepatoma.
\end{abstract}

Keywords: apoptosis; autophagy; hepatoma; Huh-7 cells; magnoflorine (MGN); proliferation

\section{Introduction}

Hepatocellular carcinoma (HCC) is one of the most common solid malignancies of the digestive tract (Hoshimoto et al., 2018; Luan et al., 2021). It ranks as the fifth most common malignancy after lung cancer and is the second most deadly tumor (Gailhouste et al., 2013; Wu et al., 2020a]. The pathogenesis of HCC is a complex process involving multiple steps and factors, including environment, genetics, and lifestyle (Bao et al., 2018). Various risk factors and toxicants, including aflatoxins, nitrosamines, and trace elements, are closely associated with the development and progression of HCC (van Rensburg et al., 1990). The incidence of HCC is high, accounting for about 55\% of patients worldwide (Jagric and Horvat, 2020). The mechanisms underlying the pathogenesis, progression, diagnosis, and treatment of $\mathrm{HCC}$ are still a major challenge in biomedical research (Li et al., 2021). Given the lack of obvious symptoms in early stages of HCC, patients are often already at an advanced stage by the time they seek medical attention (Shun et al., 2008). Yet, traditional treatment methods, such as surgical resection, radiotherapy, and chemotherapy, have limited effects (Shun et al., 2008). In order to better treat advanced HCC, there is still a requirement to develop new and more effective therapeutic targets.

Magnoflorine (MGN), also named as thalictrine, occurs naturally in the root of buttercup plant, and can be produced by chemical synthesis as well. It has neuropsychopharmacological, antianxiety, immunomodulatory, anti-inflammatory, antioxidant, and antifungal activities (Chang et al., 2020; Okon et al., 2020a, 2020b). MGN ameliorates inflammation and fibrosis in rats 
with diabetic nephropathy via regulating the stability of LSD3A (Chang et al., 2020). MGN inhibited the proliferation of breast cancer, gastric cancer, and rhabdomyosarcoma cells in a dose-dependent manner as well as induced cell apoptosis and blocked cell cycle (Okon et al., 2020a, 2020b; Sun et al., 2020c]. Studies have shown that magnolina induces cell autophagy, cell apoptosis, and cell cycle arrest to inhibit the development of gastric cancer (Wang et al., 2020). MGN can also induce apoptosis and autophagy of breast cancer cells through Akt/mTOR signaling pathway (Wei et al., 2020). However, its possible effects on the progression of $\mathrm{HCC}$ are still indefinite.

The PI3K/Akt/mTOR pathway is closely associated with cell proliferation, apoptosis, autophagy, and inflammation (Sun et al., 2020b). mTOR is an important downstream regulator in the PI3K/Akt pathway, and plays a crucial role in protein synthesis and autophagy (GolobSchwarzl et al., 2017; Seshadri, 2021). Increasing evidences have suggested that several factors can inhibit progression of HCC through inactivation of PI3K/Akt/ mTOR signaling pathway (Golob-Schwarzl et al., 2017). In this study, the effects of MGN on the progression of Huh-7 cell proliferation, autophagy, and apoptosis were determined in vitro.

\section{Materials and Methods}

\section{Antibodies and drugs}

Anti-glyceraldehyde 3-phosphate dehydrogenase (GAPDH) antibody (1:3,000, ab8245; Abcam, Cambridge, MA, USA), LC3B antibody (1:1,000 dilution, \#4108; Cell Signaling Technology (CST) Inc., Beverly, MA, USA), Beclin-1 antibody (1:500 dilution, \#3495; CST), P62 antibody (1:1,000 dilution, ab207305; Abcam). p-PI3K (1:1,000, ab278545; Abcam), PI3K (1:1,000, ab154598; Abcam), p-AKT (1:1,000, ab38449; Abcam), AKT (1:1,000, ab8505; Abcam). p-mTOR (1:500, ab137133; Abcam), mTOR (1:1,000, ab134903; Abcam). Caspase 3 (1:1,000, ab32351; Abcam), Caspase 9 (1:1,000, ab32359; Abcam), cleaved caspase 3 (1:1,000, ab32042; Abcam), cleaved caspase 9 (1:1,000, ab2324; Abcam), and Bcl-xl (1:500, ab137133; Abcam). Magnoflorine (CAS 2141-09-5) was purchased from ChemGenes Corp (MA, USA) and dissolved in dimethyl sulfoxide (DMSO). The final concentrations of MGN in Huh-7 cell line were $0,20,40$, and $80 \mu \mathrm{M}$.

\section{Cell culture and transfection}

The human normal hepatoma cell line, Huh-7, was purchased from ATCC and maintained in Dulbecco's modified eagle medium (DMEM) supplemented with 10\% fetal bovine serum (FBS) and incubated at $37^{\circ} \mathrm{C}$ in a $5 \%$ $\mathrm{CO}_{2}$ incubator. MGN was administrated into Huh-7 cell line at the concentrations of $0,20,40$, and $80 \mu \mathrm{M}$ for $24 \mathrm{~h}$.

\section{Cell counting kit-8 (CCK-8) assay}

Huh-7 cells were plated in 96-well plates with 1,000 cell/ well density and maintained for $72 \mathrm{~h}$ upon the indicated treatment. Cells were then incubated with CCK- 8 for $4 \mathrm{~h}$ and the OD value was measured at $0,24,38$, and $72 \mathrm{~h}$ at 490-nm wave length.

\section{Immunoblot assay}

The immunoblot assay was performed according to the study conducted by Wei et al. (2020). Huh-7 cells were lysed with radioimmunoprecipitation assay (RIPA) buffer. Cells in different groups were isolated and the proteins were separated by $10 \%$ sodium dodecyl sulfate-polyacrylamide gel electrophoresis (SDS-PAGE). Proteins were then transferred onto polyvinylidene difluoride (PVDF) membranes and blocked with 5\% fat-free milk. Membranes were incubated with both primary antibodies for $1.5 \mathrm{~h}$ and secondary antibodies for another $1 \mathrm{~h}$. Signals were detected using an enhanced chemiluminescent (ECL) kit.

\section{Enzyme-linked-immunosorbent serologic assay (ELISA)}

ELISA was performed according to the study conducted by Wei et al. (2020).The effects of MGN on the activities of caspase-3 (ab252897; Abcam) and caspase-9 (ab219915; Abcam) in Huh-7 cells were detected according to the imparted instructions.

\section{Cell cycle and apoptosis assays}

For apoptosis assay, hepatoma cells were fixed with 70\% ethyl alcohol for $24 \mathrm{~h}$ at $-20^{\circ} \mathrm{C}$ and treated with Annexin $\mathrm{V}$-fluorescein isothiocyanate (FITC) and propidium iodide (PI) for $20 \mathrm{~min}$, then analyzed using a flow cytometer. For cell cycle assay, Huh-7 cells were fixed with $70 \%$ ethyl alcohol for $24 \mathrm{~h}$ at $-20^{\circ} \mathrm{C}$ and incubated with PI for $20 \mathrm{~min}$. The percentage of cells was analyzed.

\section{Statistics}

GraphPad 6.0 was used for analysis. Data were shown as mean \pm standard error of mean (SEM). One-way ANOVA was used for data comparison, and $P<0.05$ was considered statistically significant. "indicates $p<0.05, " p$ $<0.01$, and ${ }^{m * n} p<0.001$, respectively. 


\section{Results}

\section{MGN suppressed the proliferation of Huh-7 cell line}

In order to uncover the possible effects of MGN on the progression of hepatoma, its effects were determined at different concentrations of $0,20,40$, and $80 \mu \mathrm{M}$ on the proliferation of hepatoma cell line Huh-7. CCK-8 assays showed that MGN treatment suppressed the proliferation of Huh-7 cells in a concentration-dependent manner (Figure 1A). The flow cytometry (FCM) assay showed that treatment of MGN led to arrest of Huh-7 cell cycle, with the increased percentage of cells at G1 phase and decreased percentage of cells at $S$ phase (Figures $1 \mathrm{~B}$ and $\mathrm{C}$ ). Therefore, MGN treatment inhibited the proliferation of Huh-7 cells through stimulating cell cycle arrest.

\section{MGN treatment induced the autophagy in Huh-7 cell line}

Further study was intended to detect the effects of MGN on the autophagy of hepatoma cells. The immunoblot assay showed that the expression of p62, a protein that can negatively regulate autophagy, was decreased after MGN treatment (Figure 2). Besides, the expression of beclin-1 was upregulated upon the treatment of MGN, suggesting the activation of autophagy (Figure 2). The ratio of LC3II:LC3I was also increased after MGN treatment, which further confirmed that MGN treatment induced autophagy in Huh-7 cells (Figure 2).

\section{MGN treatment stimulated the apoptosis of Huh-7 cell line}

The previous results detected the effects of MGN on the apoptosis of hepatoma cells by FCM assay. Interestingly, MGN treatment significantly stimulated the apoptosis of Huh-7 cells in a concentration-dependent manner (Figures 3A and B). ELISA suggested that both caspase-3 and caspase- 9 were dramatically activated after MGN treatment in Huh-7 cells (Figure 3C). Immunoblot assay was performed to detect the protein expressions of apoptosis-related proteins upon MGN treatment at various concentrations $(20,40$, and $80 \mu \mathrm{M})$. The results showed the up-regulation of cleaved caspase- 3 and cleaved caspase-9, and the down-regulation of Bcl-xl upon MGN treatment, confirming the activation of caspase (Figure 3D). Therefore, treatment of MGN stimulated the apoptosis of Huh-7 cells.

\section{MGN treatment inactivated PI3K/AKT/mTOR pathway in Huh-7 cell line}

It is well accepted that cell proliferation, autophagy, and apoptosis could be mediated by PI3K/AKT pathway. Therefore, the involvement of PI3K/AKT pathway in the regulatory roles of $\mathrm{MGN}$ in hepatoma cell proliferation, autophagy, and apoptosis was validated. The immunoblot assay showed that MGN treatment suppressed the phosphorylated levels of PI3K and AKT in Huh-7 cells (Figure 4).
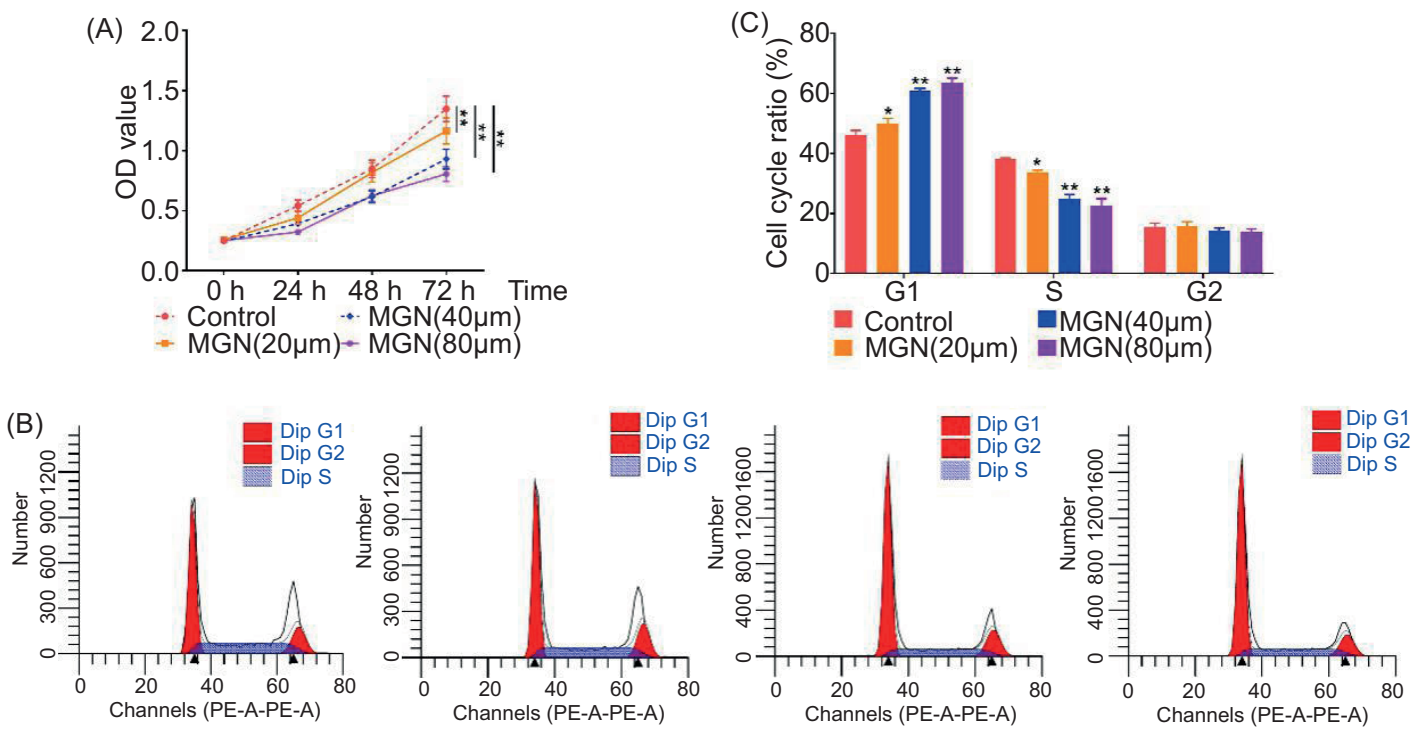

Figure 1. Magnoflorine suppressed the proliferation of Huh-7 cell line. (A) CCK-8 assay demonstrated the effects of MGN treatment on the proliferation of Huh-7 cells at 0-, 20-, 40-, and 80- $\mu \mathrm{M}$ concentrations, and the OD value at 490-nm wavelength was quantified. (B) FCM assay demonstrated the effects of MGN treatment on Huh-7 cell cycle at 0-, 20-, 40-, and 80- $\mu \mathrm{M}$ concentrations. (C) The quantification of (B). Data were presented as mean $\pm \mathrm{SEM} ;{ }^{*} P<0.05,{ }^{* *} P<0.01$. 


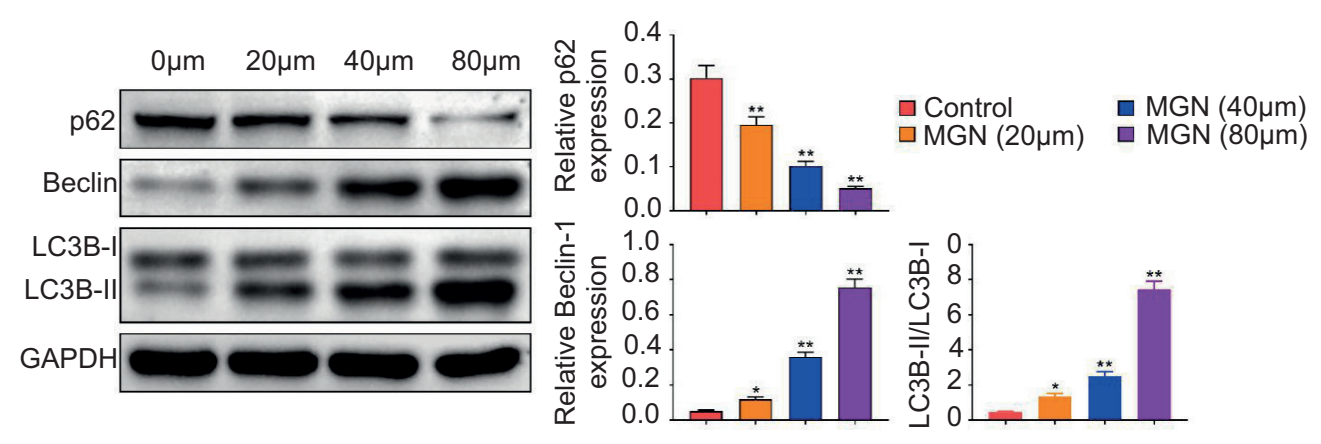

Figure 2. Magnoflorine treatment induced the autophagy of Huh-7 cell line. Immunoblot assay demonstrated the expression of proteins on Huh-7 cells after MGN treatment at 0-, 20-, 40-, and 80- $\mu \mathrm{M}$ concentrations. Data were presented as mean \pm SEM; ${ }^{*} P<0.05,{ }^{* *} P<0.01$.

(A)

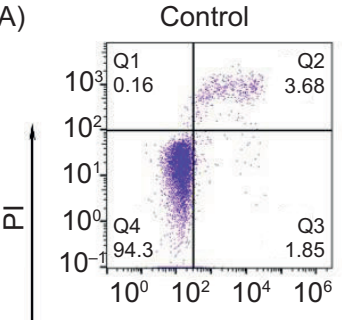

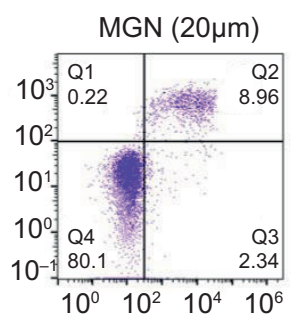

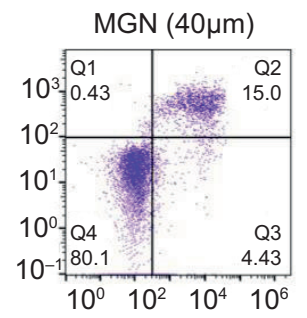

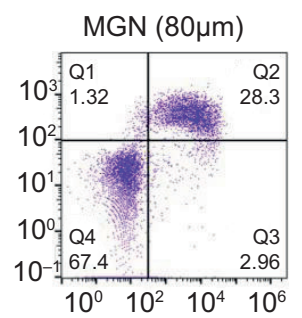

FITC
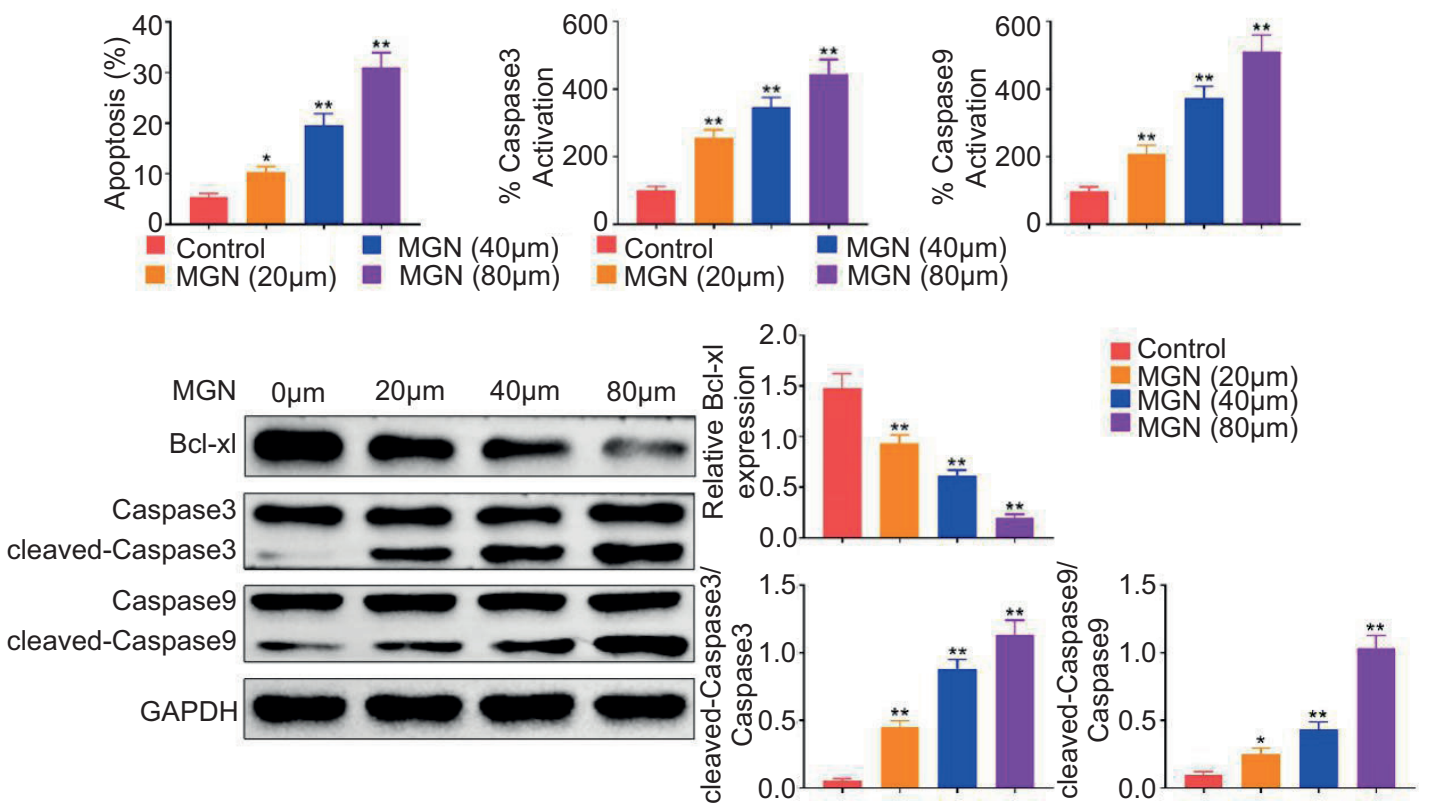

Figure 3. Magnoflorine treatment stimulated the apoptosis of Huh-7 cell line. (A) FCM assay demonstrated the effects of

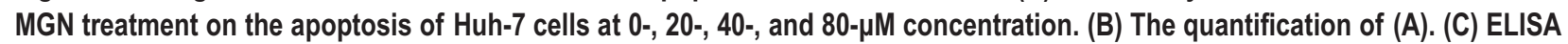
demonstrated the activities of Caspase-3 and Caspase-9 in Huh-7 cells on MGN treatment at 0-, 20-, 40-, and 80- $\mathrm{MM}$ concentrations. (D) Immunoblot assay demonstrated the expression levels of proteins on Huh-7 cells after MGN treatment at 0-, 20-, 40-, and $80-\mu \mathrm{M}$ concentrations. Data were presented as mean $\pm \mathrm{SEM} ;{ }^{*} P<0.05,{ }^{* *} P<0.01$. 

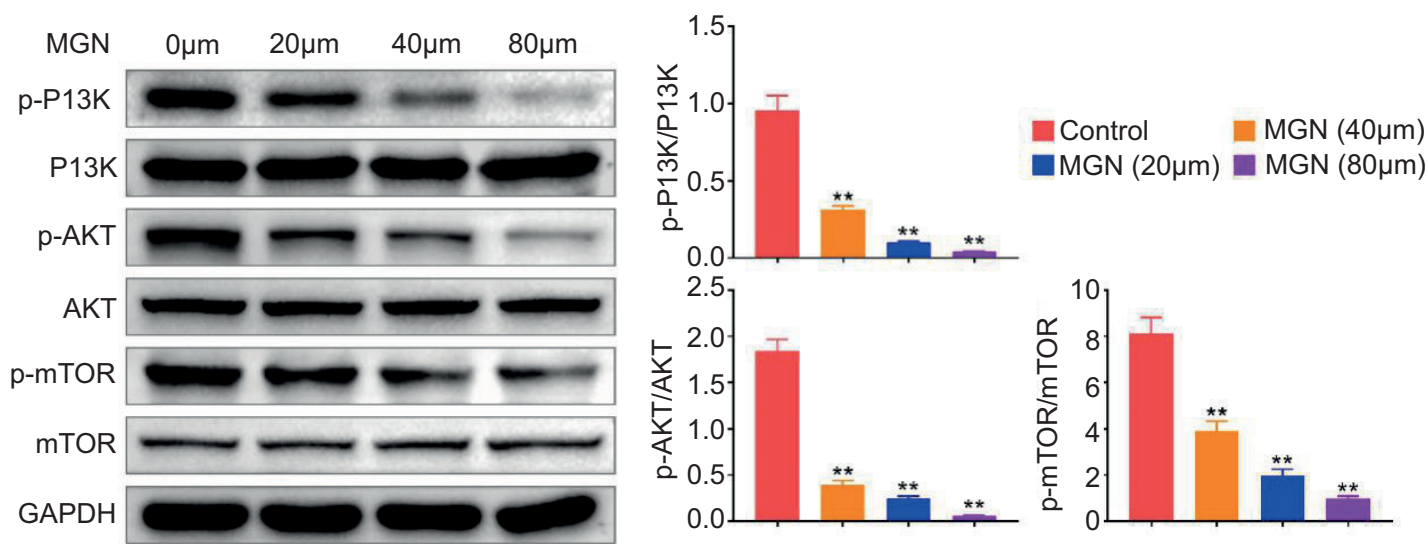

Figure 4. Magnoflorine treatment inactivated the PI3K/AKT/mTOR pathway in Huh-7 cell line. Immunoblot assay demonstrated the expression levels of indicated proteins in Huh-7 cells after MGN treatment at 0-, 20-, 40-, and 80- $\mu$ M concentrations. Data were presented as mean $\pm \mathrm{SEM}$; ${ }^{* *} P<0.01$.

In addition, the phosphorylated level of mTOR was also decreased after MGN treatment in Huh-7 cells (Figure 4). Therefore, MGN treatment inactivated PI3K/AKT/mTOR pathway in Huh-7 cells.

\section{Discussion}

In the present study, effects of MGN were revealed on the progression of Huh-7 cell proliferation, autophagy, and apoptosis. The results established that MGN could serve as a promising drug for HCC treatment. HCC is a malignant tumor with a high rate of morbidity, metastasis, and recurrence (van Rensburg et al. 1990). Although its etiology and pathogenesis have not been well explored, with early diagnosis and treatment of primary hepatoma, the overall outcomes have improved significantly (Hoshimoto et al., 2018). However, in advanced HCC, surgical resection, radiotherapy, and chemotherapy are not effective in achieving a satisfactory outcome (Galicia-Moreno et al., 2021). Even after radical resection of HCC, $\sim 60-70 \%$ patients still develop metastasis and recurrence within 5 years (Bao et al., 2018; Nurili et al., 2021). Urgent requirement is sought to find new and more effective drugs for the treatment of HCC. Here, a quaternary ammonium alkaloid, MGN, was considered a potential factor, based on its effects on the proliferation, autophagy, and apoptosis of hepatoma cells in vitro.

CCK-8 and FCM assays demonstrated that MGN suppressed the proliferation of hepatoma cells by stimulating cell cycle arrest. Immunoblot assay validated that MGN could affect the autophagy of hepatoma cells. FCM assay and ELISA suggested that MGN induced hepatoma cell apoptosis. These findings confirmed the antitumor role of MGN in hepatoma. MGN had multiple biological traits, such as antitumor, anti-inflammatory, and antioxidant activities (Chang et al., 2020; Liang et al., 2020; Okon et al., 2020b). For example, MGN had the potential to treat 2,4-Dinitrochlorobenzene (DNCB)induced atopic dermatits by suppressing the apoptosis of keratinocyte (Wu et al., 2020b). MGN also suppressed mitogen-activated protein kinase (MAPK) and nuclear factor kappa B (NF-kB) pathway to suppress inflammatory osteolysis induced by titanium particles (Sun, et al., 2020a). Importantly, MGN suppressed the progression of multiple types of cancers (Sun et al., 2020c; Wei et al., 2020). MGN also inhibited progression of gastric cancer by inducing autophagy, apoptosis, and cell cycle arrest by c-Jun $\mathrm{N}$-terminal kinase (JNK) activation regulated by reactive oxygen species (ROS) (Seshadri, 2021). These studies, together with our findings, confirmed that MGN could be an effective drug for treating hepatoma.

It is also noticed the PI3K/AKT/mTOR pathway mediated the proliferation, apoptosis, and autophagy of hepatoma cells. In fact, PI3K/AKT/mTOR pathway is known to be related with the progression of various cancers (Golob-Schwarzl et al., 2017; Guo et al., 2021). For example, YTHDF1 could promote HCC via activating $\mathrm{PI} 3 \mathrm{~K} / \mathrm{AKT} / \mathrm{mTOR}$ pathway and inducing epithelium to mesenchymal transition (Luo et al., 2021). Brucine promoted the apoptosis and suppressed the proliferation of cervical cancer cells by PI3K/AKT/mTOR pathway (Seshadri, 2021). Long non-coding RNAs (LncRNAs), such as microRNA-19b, could affect oxaliplatin chemoresistance in colon cancer through $\mathrm{PI} 3 \mathrm{~K} / \mathrm{AKT} / \mathrm{mTOR}$ pathway (Fan et al., 2021). These studies, together with our findings, confirmed that PI3K/AKT/mTOR pathway could serve as a promising therapeutic target for cancer treatment. In fact, PI3K/AKT/mTOR signaling pathway could also regulate cell migration and invasion. Therefore, MGN may also participate in progression of HCC through regulating cell migration, invasion, and 
motility through PI3K/AKT/mTOR signaling pathway, which needs further validation. A previous study has revealed that MGN improved the sensitivity of breast cancer cells to doxorubicin (DOX) by inducing apoptosis and autophagy through AKT/mTOR pathway (Wei et al., 2020). Consistently, the regulatory role of MGN in AKT/ mTOR pathway was observed in Huh-7 cells.

\section{Conclusion}

The effects of MGN on the progression of hepatoma in vitro were established through by suppressing proliferation, inducing autophagy, and stimulating apoptosis in HCC cells. Mechanically, MGN could regulate the PI3K/ AKT/mTOR pathway, and therefore affect Huh-7 cells in vitro. MGN could thus act as a promising therapeutic drug for treating hepatoma treatment.

\section{Competing Interests}

The authors state that there was no conflict of interest to disclose.

\section{Author Contributions}

Jifan $\mathrm{Xu}$ and $\mathrm{Bo} \mathrm{Du}$ designed the study and supervised data collection. Yunfeng Liu analyzed and interpreted the data. Chonglin Tao prepared and reviewed the draft of manuscript for publication. All authors read and approved the final manuscript.

\section{References}

Bao T., Ke Y., Wang Y., Wang W., Li Y., Wang Y., Kui X., Zhou Q., Zhou H., and Zhang C., 2018. Taraxasterol suppresses the growth of human liver cancer by upregulating Hint1 expression. Journal of Molecular Medicine (Berlin) 96(7): 661-672. https:// doi.org/10.1007/s00109-018-1652-7. PMID: 29806073.

Chang L., Wang Q., Ju J., Li Y., Cai Q., Hao L. and Zhou Y., 2020. Magnoflorine ameliorates inflammation and fibrosis in rats with diabetic nephropathy by mediating the stability of lysine-specific demethylase 3A. Frontiers in Physiology 11: 580406. https://doi. org/10.3389/fphys.2020.5804.06. PMID: 33414721.

Fan Y., Zeng F., Ma L., and Zhang H., 2021. Effects of beta-carboline alkaloids from Peganum harmala on the FAK/PI3K/AKT/ M-tor pathway in human gastric cancer cell line SGC-7901 and tumor-bearing mice. Pakistan Journal of Pharmaceutical Sciences. 34(3): 891-898. PMID: 34602411.

Gailhouste L., Gomez-Santos L. and Ochiya T., 2013. Potential applications of miRNAs as diagnostic and prognostic markers in liver cancer. Frontiers in Bioscience-Landmark 18: 199-223. https://doi.org/10.2741/4096. PMID: 23276918.
Galicia-Moreno M., Silva-Gomez J.A., Lucano-Landeros S., Santos A., Monroy-Ramirez H.C. and Armendariz-Borunda J., 2021. Liver cancer: therapeutic challenges and the importance of experimental models. Canadian Journal of Gastroenterology and Hepatology 8837811. https://doi.org/10.1155/2021/8837811. PMID: 33728291.

Golob-Schwarzl N., Krassnig S., Toeglhofer A.M., Park Y.N., GoggKamerer M., Vierlinger K., Schroder F., Rhee H., Schicho R., Fickert P., and Haybaeck J., 2017. New liver cancer biomarkers: $\mathrm{PI} 3 \mathrm{~K} / \mathrm{AKT} / \mathrm{mTOR}$ pathway members and eukaryotic translation initiation factors. The European Journal of Cancer 83: 56-70. https://doi.org/10.1016/j.ejca.2017.06.003. PMID: 28715695.

Guo C., Chu H., Gong Z., Zhang B., Li C., Chen J. and Huang L., 2021. HOXB13 promotes gastric cancer cell migration and invasion via IGF-1R upregulation and subsequent activation of PI3K/AKT/mTOR signaling pathway. Life Sciences 278: 119522. https://doi.org/10.1016/j.lfs.2021.119522. PMID: 33894267.

Hoshimoto S., Hoshi N., Ozawa I., Tomikawa M., Shirakawa H., Fujita T., Wakamatsu S., Hoshi S., Hirabayashi K., Hishinuma S. Hirabayashi K., and Hishinuma S., 2018. Rapid progression of a granulocyte colony-stimulating factor-producing liver tumor metastasized from esophagogastric junction cancer: a case report and literature review. Oncology Letters 15(5): 6475-6480. https://doi.org/10.3892/ol.2018.8144. PMID: 29725401.

Jagric T. and Horvat M., 2020. Surgical resection of synchronous liver metastases in gastric cancer patients. A propensity scorematched study. Radiology and Oncology 55(1): 57-65. https:// doi.org/10.2478/raon-2020-0067. PMID: 33885239.

Li Z.Y., Li H.L., Ji X.W., Shen Q.M., Wang J., Tan Y.T. and Xiang Y.B., 2021. Dose-response association between adiposity and liver cancer incidence: a prospective cohort study among non-smoking and non-alcohol-drinking Chinese women. Cancer Epidemiology Biomarkers \& Prevention 30(6): 12001207. https://doi.org/10.1158/1055-9965.EPI-20-1610. PMID: 33849965.

Liang X., Xiang Y., Li Y., Feng P., Qin Y. and Lai X., 2020. A rapid method for simultaneous quantification of berberine, berbamine, magnoflorine and berberrubine in mouse serum using UPLC-MS/MS. Journal of Chromatography B: Analytical Technologies in the Biomedical and Life Sciences 1142: 122040. https://doi.org/10.1016/j.jchromb.2020.122040. PMID: 32145638.

Luan Y., Li M., Zhao Y., Li Q., Wen J., Gao S. and Yang Y., 2021. Centrosomal-associated proteins: potential therapeutic targets for solid tumors? Biomedicine \& Pharmacotherapy 144: 112292. https://doi.org/10.1016/j.biopha.2021.112292. PMID: 34700231.

Luo X., Cao M., Gao F. and He X., 2021. YTHDF1 promotes hepatocellular carcinoma progression via activating $\mathrm{PI} 3 \mathrm{~K} / \mathrm{AKT} / \mathrm{mTOR}$ signaling pathway and inducing epithelial-mesenchymal transition. Experimental Hematology \& Oncology 10(1): 35. https:// doi.org/10.1186/s40164-021-00227-0. PMID: 34088349.

Nurili F., Monette S., Michel A.O., Bendet A., Basturk O., Askan G., Cheleuitte-Nieves C., Yarmohammadi H., Maxwell A.W.P., and Ziv E., 2021. Transarterial embolization of liver cancer in a transgenic pig model. Journal of Vascular and Interventional Radiology 32(4): 510-517 e513. https://doi.org/10.1016/j. jvir.2020.09.011. PMID: 33500185. 
Okon E., Luszczki J.J., Kukula-Koch W., Halasa M., Jarzab A., Khurelbat D., Stepulak A. and Wawruszak A., 2020a. Synergistic or additive pharmacological interactions between magnoflorine and cisplatin in human cancer cells of different histological origin. International Journal of Molecular Sciences 21(8). https:// doi.org/10.3390/ijms21082848. PMID: 32325867.

Okon E., Kukula-Koch W., Halasa M., Jarzab A., Baran M., Dmoszynska-Graniczka M., Angelis A., Kalpoutzakis E., Guz M., Stepulak A et al., 2020b. Magnoflorine-isolation and the anticancer potential against NCI-H1299 lung, MDA-MB-468 breast, T98G glioma, and TE671 rhabdomyosarcoma cancer cells. Biomolecules 10(11). https://doi.org/10.3390/biom10111532. PMID: 33182753.

Seshadri V.D. 2021. Brucine promotes apoptosis in cervical cancer cells (ME-180) via suppression of inflammation and cell proliferation by regulating PI3K/AKT/mTOR signaling pathway. Environmental Toxicology https://doi.org/10.1002/tox.23304. PMID: 34076332.

Shun S.C., Chiou J.F., Lai Y.H., Yu P.J., Wei L.L., Tsai J.T., Kao C.Y. and Hsiao Y.L., 2008. Changes in quality of life and its related factors in liver cancer patients receiving stereotactic radiation therapy. Support Care Cancer 16(9): 1059-1065. https://doi. org/10.1007/s00520-007-0384-y. PMID: 18197433.

Sun Z., Zeng J., Wang W., Jia X., Wu Q., Yu D. and Mao Y., 2020a. Magnoflorine suppresses MAPK and NF-kappa B signaling to prevent inflammatory osteolysis induced by titanium particles in vivo and osteoclastogenesis via RANKL in vitro. Frontiers in Pharmacology 11: 389. https://doi.org/10.3389/ fphar.2020.00389. PMID: 32300300.

Sun R., Zhai R., Ma C. and Miao W., 2020b. Combination of aloin and metformin enhances the antitumor effect by inhibiting the growth and invasion and inducing apoptosis and autophagy in hepatocellular carcinoma through PI3K/AKT/mTOR pathway.
Cancer Medicine 9(3): 1141-1151. https://doi.org/10.1002/ cam4.2723. PMID: 31830378.

Sun X.L., Zhang X.W., Zhai H.J., Zhang D. and Ma S.Y., 2020c. Magnoflorine inhibits human gastric cancer progression by inducing autophagy, apoptosis and cell cycle arrest by JNK activation regulated by ROS. Biomedicine \& Pharmacotherapy 125: 109118. https://doi.org/10.1016/j.biopha.2019.109118. PMID: 32106366.

van Rensburg S.J., van Schalkwyk G.C. and van Schalkwyk D.J., 1990. Primary liver cancer and aflatoxin intake in Transkei. Journal of Environmental Pathology, Toxicology, and Oncology 10(1-2): 11-16. PMID: 2231312.

Wang Y., Shang G., Wang W., Qiu E., Pei Y. and Zhang X., 2020. Magnoflorine inhibits the malignant phenotypes and increases cisplatin sensitivity of osteosarcoma cells via regulating miR410-3p/HMGB1/NF-kappaB pathway. Life Sciences 256: 117967. https://doi.org/10.1016/j.lfs.2020.117967. PMID: 32553931.

Wei T., Xiaojun X. and Peilong C., 2020. Magnoflorine improves sensitivity to doxorubicin (DOX) of breast cancer cells via inducing apoptosis and autophagy through AKT/mTOR and p38 signaling pathways. Biomedicine \& Pharmacotherapy 121: 109139. https://doi.org/10.1016/j.biopha.2019.109139. PMID: 31707337.

Wu Y., Matsumoto K., Chen Y.M., Tung Y.C., Chiu T.Y. and Hasegawa T., 2020a. Comparison of the cost of illness of primary liver cancer between Japan and Taiwan. Health Economics Review 10(1): 38. https://doi.org/10.1186/s13561-020-00296-7. PMID: 33280073.

Wu S., Yu D., Liu W., Zhang J., Liu X., Wang J., Yu M., Li Z., Chen Q., Li X., Ye X., 2020b. Magnoflorine from coptis Chinese has the potential to treat DNCB-induced atopic dermatits by inhibiting apoptosis of keratinocyte. Bioorganic \& Medicinal Chemistry 28(2): 115093. https://doi.org/10.1016/j.bmc.2019.115093. PMID: 31859028. 